\title{
Double adrenocortical adenomas harboring independent KCNJ5 and PRKACA somatic mutations
}

\author{
Kazutaka Nanba', Kei Omata', Scott A Tomlins ${ }^{2,3,4,5}$, Thomas J Giordano ${ }^{2,3,6}$, \\ Gary D Hammer ${ }^{1,6,7}$, William E Rainey ${ }^{1}$ and Tobias Else ${ }^{6}$ \\ 'Departments of Molecular and Integrative Physiology \& Internal Medicine, ${ }^{2}$ Department of Pathology, \\ ${ }^{3}$ Comprehensive Cancer Center, ${ }^{4}$ Michigan Center for Translational Pathology, ${ }^{5}$ Department of Urology, \\ ${ }^{6}$ Division of Metabolism, Endocrinology, and Diabetes and ${ }^{7}$ Endocrine Oncology Program, Center for \\ Organogenesis, University of Michigan, Ann Arbor, Michigan, USA
}

\author{
Correspondence \\ should be addressed \\ to T Else \\ Email \\ telse@umich.edu
}

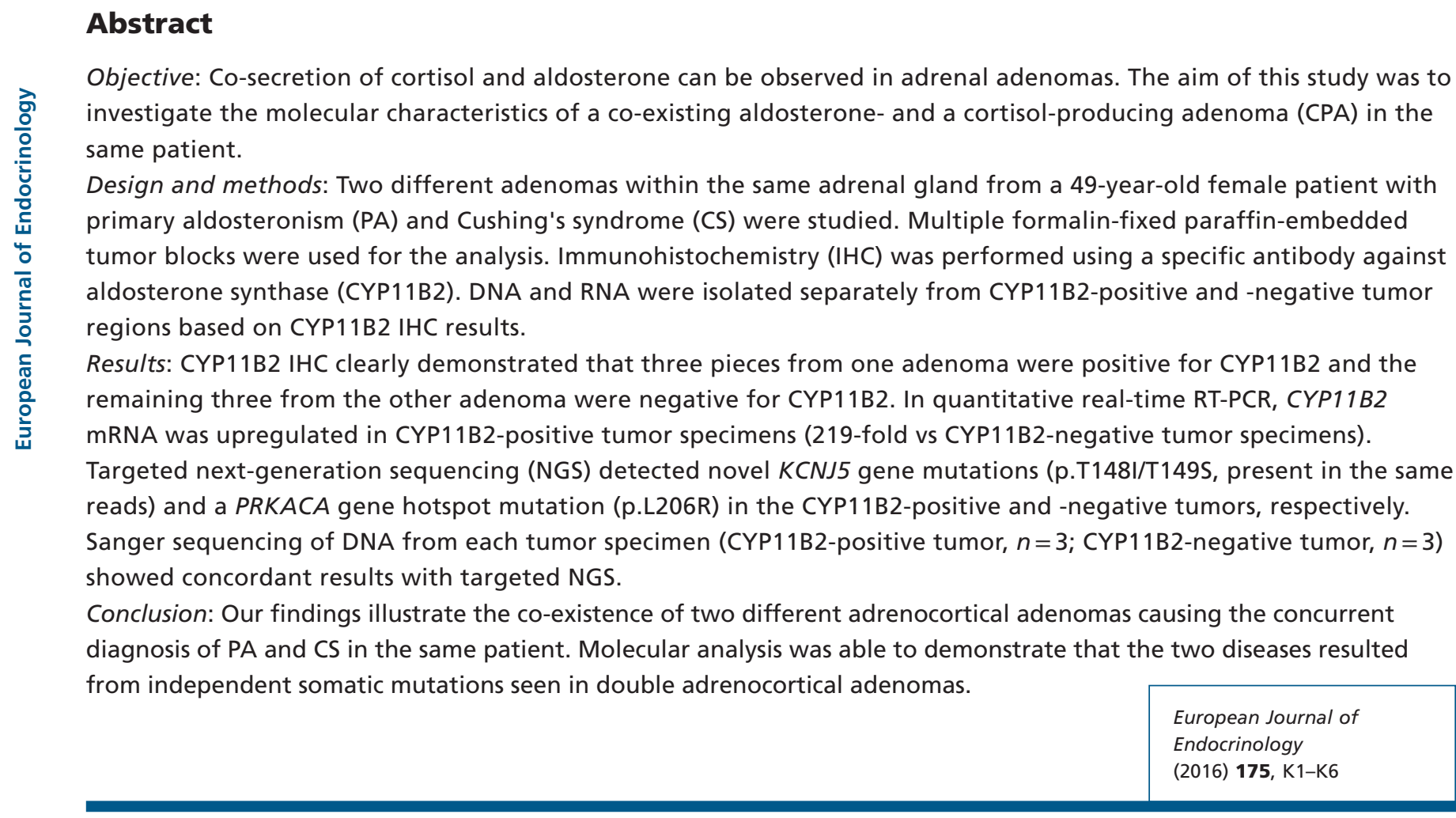

Introduction

Primary aldosteronism (PA) is a common form of secondary hypertension caused by autonomous adrenal aldosterone production. PA is clinically characterized by hypertension and hypokalemia. Aldosterone-producing adenoma (APA) and idiopathic hyperaldosteronism are the major subtypes of PA. Adrenal Cushing syndrome (CS) is caused by glucocorticoid excess due to a cortisol- producing adenoma (CPA), resulting in hypertension, obesity, type 2 diabetes, and vertebral osteoporosis. As a result of cortisol excess, CS patients present with unique clinical features such as easy bruising, skin atrophy, facial plethora, striae, and dorsocervical fat pad. Over the last decades, an increasing number of patients with subclinical Cushing syndrome (SCS) have been identified. SCS is often
() 2016 European Society of Endocrinology Printed in Great Britain
Published by Bioscientifica Ltd. 
characterized by mild hypercortisolism without specific signs or symptoms for CS. In patients with PA, concurrent autonomous cortisol production has been increasingly recognized (1). The majority of the reported cases have had SCS, whereas overt CS has been rarely found.

Next-generation sequencing (NGS) has identified several somatic and germline mutations underlying sporadic APA and/or familial PA, such as mutations in an inwardly rectifying potassium channel (KCNJ5) (2), the P-type ATPase gene family (ATP1A1 and ATP2B) (3), an L-type voltage-gated calcium channel $(C A C N A 1 D)(4,5)$, and T-type voltage-gated calcium channel $(C A C N A 1 H)(6)$. These mutations alter the pathway involved in regulation of aldosterone production, such as potassium sensing, membrane potential regulation, intracellular $\mathrm{Ca}^{2+}$ levels, and steroidogenesis. More recently, recurrent activating mutations in PRKACA, encoding the catalytic subunit $\alpha$ of protein kinase A (PKA) have been identified in CPA (7). PRKACA gene mutations are found in $35-65 \%$ of the patients with CS (8). Herein, we report a case of PA and CS due to the co-existence of two adenomas harboring novel KCNJ5 somatic mutations and a PRKACA somatic mutation.

\section{Patient and methods}

\section{Case report}

The patient was a 49-year-old African-American woman referred for further evaluation of endocrine hypertension. Her past medical history was positive for a pregnancyassociated deep venous thrombosis and her family history was negative for any adrenal or endocrine diseases. She had a 5-year history of high blood pressure and 3 years of hypokalemia necessitating replacement doses of $30-90 \mathrm{mmol} / \mathrm{day}$ of potassium. Over the course of the recent year, she had a weight gain of $27 \mathrm{~kg}$ (weight at presentation $154 \mathrm{~kg}$ ). She noted some facial fullness and rounding and had recently been diagnosed with prediabetes. She complained of depression, easy bruising, and muscle weakness, particularly while climbing stairs. On examination, she was found to be obese with facial rounding and increased prominence of the supraclavicular fat pads. Blood pressure was $166 / 97 \mathrm{mmHg}$. There were some pale striae on the abdomen, no skin atrophy, but some acanthosis of the neck. Laboratory evaluation revealed an aldosterone of $45 \mathrm{ng} / \mathrm{dL}(1248 \mathrm{pmol} / \mathrm{L})$ $(4-31 \mathrm{ng} / \mathrm{dL})$, a renin of $0.4 \mathrm{ng} / \mathrm{mL} / \mathrm{h}(0.11 \mathrm{ng} / \mathrm{L} \cdot \mathrm{s})(1-7 \mathrm{ng} /$ $\mathrm{mL} / \mathrm{h})$, and a $24 \mathrm{~h}$ urine cortisol of $47.3 \mu \mathrm{g} / 24 \mathrm{~h}(130.5 \mathrm{nmol} /$ day) $(20-90 \mu \mathrm{g} / 24 \mathrm{~h})$, with a low morning adrenocorticotrophic hormone (ACTH) of $<9 \mathrm{pg} / \mathrm{mL}(1.98 \mathrm{pmol} / \mathrm{L})$
$(9-52 \mathrm{pg} / \mathrm{mL})$. CT showed two adrenocortical adenomas in the right adrenal $(2.7$ and $3.0 \mathrm{~cm})$. The posterior mass measured 4 Hounsfield Unit (HU) on unenhanced CT scan (lipid-rich adenoma), and the anterior mass measured $20 \mathrm{HU}$, with a percentage enhancement washout value of 63\% (lipid poor adenoma) (9) (Fig. 1A, B and C). NP59 scan showed suppressed uptake in the contralateral gland. Both lesions were identified in the pathological specimen and the diagnosis was that of an adrenal adenoma in both masses. Following surgery, the patient felt significant mood improvement and blood pressure and potassium levels normalized. She lost $6 \mathrm{~kg}$ over the course of 3 months following surgery. She was diagnosed with adrenal insufficiency following surgery with stimulation to maximum of $1.4 \mu \mathrm{g} / \mathrm{dL}(38.6 \mathrm{nmol} / \mathrm{L})(>18 \mu \mathrm{g} / \mathrm{dL})$ cortisol and did require glucocorticoid replacement therapy.

\section{Materials}

Multiple formalin-fixed paraffin-embedded (FFPE) tumor blocks were used for the analysis. This study was approved by Institutional Review Board of the University of Michigan (HUM00042749).

\section{Immunohistochemistry}

Immunohistochemistry (IHC) was performed using antibodies against aldosterone synthase (CYP11B2) (mouse monoclonal; 1:1500, Millipore, \#MABS1251) (10) and cytochrome P450 17A1 (CYP17) (mouse monoclonal; 1:500, kindly provided by Dr Richard Parker, University of Alabama at Birmingham) as described previously (11).

\section{DNA/RNA isolation}

For each sample, six unstained FFPE slides were used for genomic DNA (gDNA) and RNA isolation. gDNA and RNA of CYP11B2-positive and -negative regions were isolated separately based on CYP11B2 IHC using the AllPrep DNA/RNA FFPE kit (Qiagen) as described previously (11).

\section{Quantitative real-time RT-PCR}

Total RNA was reverse transcribed using the High-Capacity cDNA Archive Kit (Applied Biosystems). Quantitative real-time RT-PCR (RT-qPCR) was performed in the ABI StepOnePlus Real-Time PCR systems (Applied Biosystems). CYP11B2 primer/probe mixtures were prepared as described previously (12). Primer/probe mixtures for the 

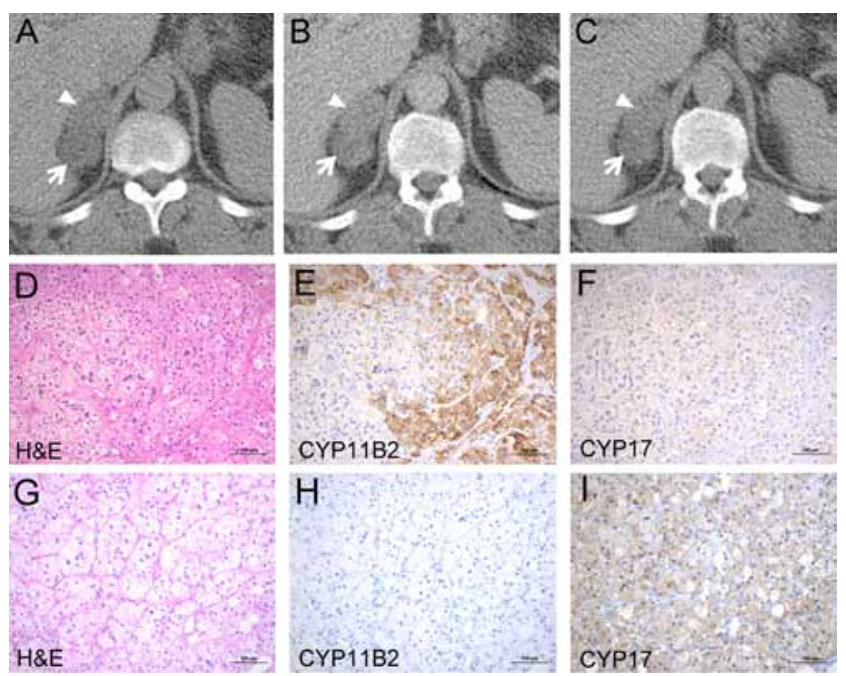

$\mathrm{H}$

CYP11B2

\section{Figure 1}

Imaging and histopatohogical findings of adrenal tumors.

( $A$, B and C) Abdominal CT showing adrenocortical adenomas in the right adrenal. (A) Unenhanced CT scan. The Hounsfield Unit (HU) of the anterior tumor (arrowhead) and the posterior tumor (arrow) were 20 and $4 \mathrm{HU}$, respectively. (B) Enhanced CT at $100 \mathrm{~s}$. The $\mathrm{HU}$ of the anterior tumor (arrowhead) and posterior tumor (arrow) were 47 and $23 \mathrm{HU}$, respectively. (C) Delayed CT at $15 \mathrm{~min}$. The HU of the anterior tumor (arrowhead) and the posterior tumor (arrow) were 30 and $14 \mathrm{HU}$, respectively. (D, E, F, G, H and I) Histopathological findings of the anterior tumor $(D, E$ and $F)$ and the posterior tumor (G, H and I). The anterior tumor showed positive staining for CYP11B2 and the posterior tumor was negative for CYP11B2 (E and H). The posterior tumor had higher expression of CYP17 compared with the anterior tumor (F and I). Scale bars $=100 \mu \mathrm{m}$. D and G, H\&E; E and H, CYP11B2 IHC; F and I, CYP17 IHC. H\&E, hematoxylin and eosin staining.

amplification of $\beta$-actin (ACTB, Hs01060665_g1) were purchased from Applied Biosystems. ACTB transcript was used for normalization of sample loading. Relative quantification was determined using comparative threshold cycle method (13).

\section{Targeted NGS}

For mutation analysis of CYP11B2-positive tumor specimen 1 (B2T1) and CYP11B2-negative tumor specimen 1 (T1) samples, 20ng of isolated gDNA was used for barcoded library generation by multiplexed PCR using a custom Ion AmpliSeq Panel and the Ion AmpliSeq Library kit 2.0 (Life Technologies) according to the manufacturer's instructions. The custom Ion AmpliSeq
Panel was designed to target genes previously shown to be mutated in APA or other adrenal hyperplasias/neoplasms. The panel contains 499 independent primer pairs targeting the entire coding regions of genes with reported germline or somatic mutations in PA (KCNJ5, ATP1A1, $A T P 2 B 3, C A C N A 1 D$, and CACNA1H), genes shown to harbor germline or somatic variants associated with adrenal hyperplasia (phosphodiesterase 11A (PDE11A), phosphodiesterase 8B (PDE8B), protein kinase, cAMPdependent, regulatory, type $1 \alpha$ (PRKAR1A), PRAKACA, and armadillo repeat containing 5 (ARMC5)), and oncogene hotspots in guanine nucleotide-binding protein subunit $\alpha(G N A S)$ and catenin $\beta 1,88 \mathrm{kDa}$ (CTNNB1). Template preparation and NGS of multiplexed templates were performed as described previously (11) using Ion 318 Chip v2 on the Ion Torrent Personal Genome Machine (PGM) Sequencer (Life Technologies). Data analysis methods for somatic variant identification are described in Supplementary methods, see section on supplementary data given at the end of this article.

\section{Direct sequencing of PCR products and subsequent analysis}

Bidirectional Sanger sequencing analyses of KCNJ5 and PRKACA gene for DNA samples isolated from each tumor piece were performed using primers as follows: KCNJ5 forward 5'-GGACCATGTTGGCGACCAAGAGTG-3', reverse 5'-GACAAACATGCACCCCACCATGAAG-3'; PRKACA forward 5'-GGTGACAGACTTCGGTTTCGC-3', reverse 5'-CCTTGTTGTAGCCCTGGAGCA-3' (14). For each PCR reaction, $20 \mathrm{ng}$ of template gDNA was used. Sanger sequencing of the purified products was undertaken on the Applied Biosystems 3730 XL sequencer. The PCR product from CYP11B2-positive specimen 3 (B2T3) DNA sample was inserted into pCR2.1-TOPO vector using a TOPO TA cloning kit (Invitrogen) for subsequent analysis. After chemical transformation using TOP10 competent cells, several carbenicillin-resistant clones were picked and sequenced.

\section{Results}

CYP11B2 IHC clearly demonstrated that the anterior adenoma showed positive staining for CYP11B2 (B2T1, B2T2 and B2T3) and the posterior adenoma was negative for CYP11B2 (T1, T2 and T3). CYP11B2positive tumor specimens exhibited positive but lower expression of CYP17 compared with the 
Table 1 Somatic mutations identified by targeted NGS. All high-confidence somatic nonsynonymous variants identified in CYP11B2-positive tumor specimen 1 (B2T1) and CYP11B2-negative tumor specimen 1 (T1) are shown. The variant allele frequency in the matched tumor specimen, that is, $\mathrm{T} 1$ for $\mathrm{B} 2 \mathrm{~T} 1$ and $\mathrm{B} 2 \mathrm{~T} 1$ for $\mathrm{T} 1$, respectively, is shown for comparison.

\begin{tabular}{|c|c|c|c|c|c|c|c|c|c|}
\hline Sample & Gene & $\begin{array}{c}\text { Reference } \\
\text { allele }\end{array}$ & $\begin{array}{l}\text { Variant } \\
\text { allele }\end{array}$ & $\begin{array}{l}\text { Amino acid } \\
\text { change }\end{array}$ & FAO & FDP & $\begin{array}{c}\text { Variant allele } \\
\text { frequency } \\
\text { (FAO/FDP; \%) }\end{array}$ & $\begin{array}{l}\text { Variant allele } \\
\text { frequency in } \\
\text { matched tumor } \\
\text { specimen }(\%)\end{array}$ & $\begin{array}{l}\text { Reference } \\
\text { sequence }\end{array}$ \\
\hline B2T1 & KCNJ5 & C & $\mathrm{T}$ & T148I & 691 & 1999 & 35 & 0 & NM_000890 \\
\hline B2T1 & KCNJ5 & $A$ & $\mathrm{~T}$ & T149S & 702 & 1997 & 35 & 0 & NM_000890 \\
\hline T1 & $P R K A C A$ & $A$ & C & L206R & 553 & 1996 & 28 & 0 & NM_002730 \\
\hline
\end{tabular}

FAO, flow-corrected variant allele-containing read; FDP, flow-corrected read depth.

CYP11B2-negative tumor specimens (Fig. 1D, E, F, $\mathrm{G}, \mathrm{H}$ and $\mathrm{I})$. In RT-qPCR, mean of CYP11B2 mRNA expression in three specimens from the CYP11B2positive adenoma was 219-fold higher than that in CYP11B2-negative adenoma, confirming accurate sample collection. Targeted NGS identified novel KCNJ5 mutations (c.443C > T and c.445A>T, p.T148I/T149S) in a specimen from the anterior adenoma (B2T1) and a PRKACA hotspot mutation (c.617T >G, p.L206R) in a specimen from the posterior adenoma (T1) (Table 1). Read level analysis in Integrative Genomics Viewer demonstrated that the KCNJ5 mutations were present on the same reads (in cis) as shown in Supplementary Fig. 1. We further investigated mutational heterogeneity through assessing multiple tumor specimens. As shown in Fig. 2A and B KCNJ5 mutations were identified in all CYP11B2-positive anterior adenoma specimens by Sanger sequencing, but not in CYP11B2-negative tumor specimens from the posterior adenoma. However, PRKACA mutations were identified only in CYP11B2negative tumor specimens from the posterior adenoma (Fig. 2A and C). Subsequent analysis of PCR product from B2T3 DNA confirmed the NGS identified KCNJ5 mutations (Fig. 2B).

\section{Discussion}

Adrenal tumors are common neoplasms and the prevalence increases with age. Most are benign cortical adenomas and some are associated with endocrine disorders such as PA and CS. Although adrenal tumors with concomitant autonomous aldosterone and cortisol secretion have been increasingly recognized, their molecular characteristics remain to be elucidated.

This study demonstrates that the adrenal tumors in our patient have different characteristics in terms of steroidogenic enzyme expression and mutation status. KCNJ5 encodes the G-protein-activated inwardly rectifying potassium channel 4 (GIRK4). Although the KCNJ5 gene mutations observed in this study have not been reported previously, two somatic mutations involving T148 and T149 located near the selectivity filter of the GIRK4 channel pore have been identified in APA (p.T148_T149insT, p.T148_T149insR) and in vitro analyses demonstrated that both mutations caused membrane depolarization, raised intracellular $\mathrm{Ca}^{2+}$, and increased aldosterone production $(15,16)$, supporting a pathological potential of the KCNJ5 mutations identified in this case (p.T148I/T149S). Additionally, considering higher conservation of T149 residue among orthologs and paralogs compared with T148 (2), the mutation altering the amino acid in position 149 likely has a more important pathophysiological role than the change in position 148. PRKACA encodes the catalytic subunit of cyclic AMP-dependent PKA. The p.L206R mutation is located in a highly conserved core of the domain responsible for the interaction between the regulatory and catalytic subunits of PKA (7). Functional experiments revealed that the mutation caused constitutive PKA activation, providing a molecular explanation for the development of $\operatorname{CPA}(7,8)$. The mutation analysis suggests that the CYP11B2-positive tumor was the main source of autonomous aldosterone production and the CYP11B2-negative and CYP17positive tumor was the main source of autonomous cortisol production in this case. It remains speculative whether the co-occurrence of two adenomas in a single patient might be caused by a common underlying genetic predisposition or simply a chance event. In most cases, the pathomechanism of hypercortisolism accompanying PA is believed to be co-secretion of cortisol and aldosterone by the same adenoma. Considering the rare co-appearance of APA and CPA in a single adrenal gland, the adenomas likely have occurred 


\section{A CYP11B2 IHC}

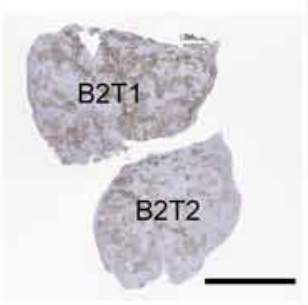

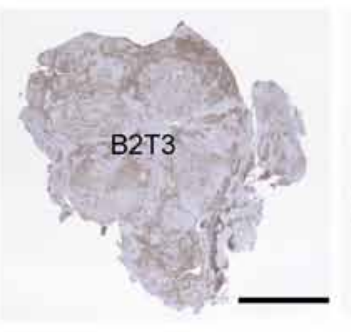
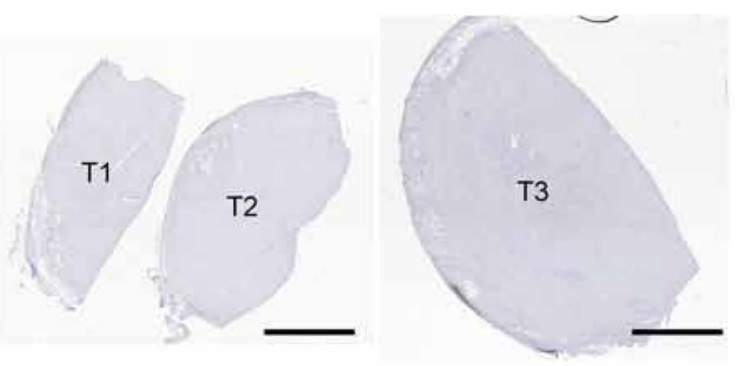

KCNJ5 (p.T148I/T149S)

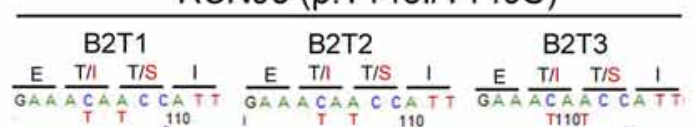

\section{B KCNJ5}
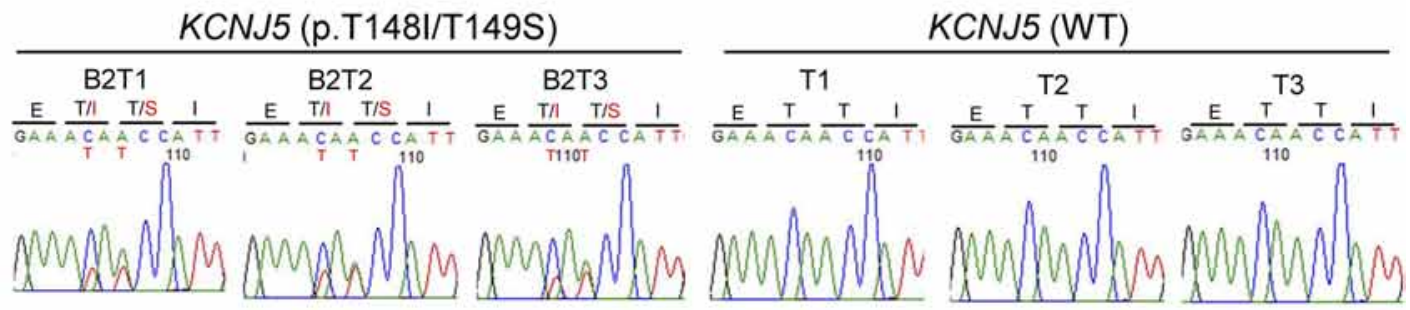

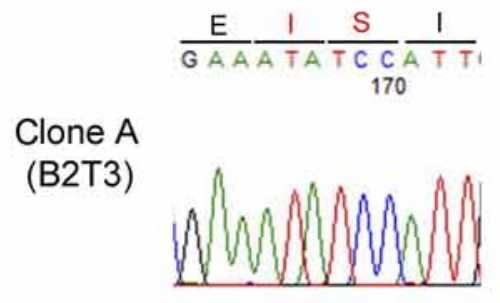

PRKACA (WT)

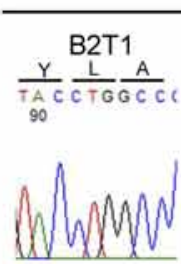

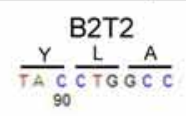

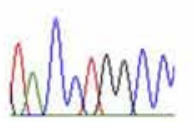

Clone B

(B2T3)

\section{PRKACA}

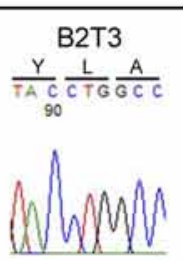

\section{Figure 2}

CYP11B2 IHC and Sanger sequencing results of each tumor piece. (A) Low magnification scan of tumor pieces with CYP11B2 IHC (CYP11B2; brown). Scale bars $=5 \mathrm{~mm}$. (B) Results of Sanger sequencing of $K C N J 5$ gene (upper panel) and subsequent analysis of PCR product (lower panel). KCNJ5 gene mutations (p.T148I/T149S) were detected in B2T1, B2T2, and B2T3, but not in T1, T2, and T3. Subsequent analysis of PCR product (B2T3) showed both mutations were present in the same allele (clone A) and the other allele was WT (clone B). (C) Results of Sanger sequencing of PRKACA gene. PRKACA gene mutations (p.L206R) were identified in T1, T2, and T3, but not in B2T1, B2T2, and B2T3. IHC, immunohistochemistry; B2T1, CYP11B2-positive tumor specimen 1; B2T2, CYP11B2-positive tumor specimen 2; B2T3, CYP11B2-positive tumor specimen 3; T1, CYP11B2-negative tumor specimen 1; T2, CYP11B2-negative tumor specimen 2; T3, CYP11B2-negative tumor specimen 3. WT, Wild type.

independently through two independent genetic events rather than having a common progenitor of the two adenomas. There remains a possible involvement of a susceptibility gene to tumor development. However, more patients and families would be necessary to attempt to define this susceptibility locus.
In this study, we demonstrate a case of PA and CS due to two different adrenocortical adenomas. This is certainly a quite rare case. However, it is important to notice any hypercortisolism in patients undergoing adrenal surgery in order to consider the necessity of perioperative hydrocortisone supplementation. This study also has 
implications for the pathologic distinction of nodular hyperplasia and double adenoma in endocrine neoplasia. Our finding of distinct mutations in discrete masses provides compelling evidence for a double adenoma model that also applies to other endocrine glands.

\section{Supplementary data}

This is linked to the online version of the paper at http://dx.doi.org/10.1530/ EJE-16-0262.

\section{Declaration of interest}

S A T has received honoraria from Thermo Fisher Scientific and had a separate sponsored research agreement with Thermo Fisher Scientific. None of the study described herein was supported by Thermo Fisher Scientific and they had no role in the data collection, interpretation, or analysis, and did not participate in the study design or the decision to submit for publication. The remaining authors have nothing to disclose.

\section{Funding}

This work was supported by grants from the NIDDK (DK106618) to W E R and S A T. T E is sponsored by American Heart Association 14SDG 17990000. K N is supported by American Heart Association 14POST20020003. S A T is supported by the A. Alfred Taubman Medical Research Institute and a National Cancer Institute Grant CA46592 (to the Michigan Cancer Center Core). This research is also supported (in part) by the National Institutes of Health through the University of Michigan's Cancer Center Support Grant (5 P30 CA46592).

\section{Acknowledgments}

The authors thank $\mathrm{Dr}$ Richard Parker (University of Alabama at Birmingham) for the generous gift of CYP17 antibody and Tina Fields for excellent immunohistochemical support.

\section{References}

1 Spath M, Korovkin S, Antke C, Anlauf M \& Willenberg HS. Aldosterone- and cortisol-co-secreting adrenal tumors: the lost subtype of primary aldosteronism. European Journal of Endocrinology 2011164 447-455. (doi:10.1530/EJE-10-1070)

2 Choi M, Scholl UI, Yue P, Bjorklund P, Zhao B, Nelson-Williams C, Ji W, Cho Y, Patel A, Men CJ et al. K+ channel mutations in adrenal aldosterone-producing adenomas and hereditary hypertension. Science 2011331 768-772. (doi:10.1126/ science.1198785)

3 Beuschlein F, Boulkroun S, Osswald A, Wieland T, Nielsen HN, Lichtenauer UD, Penton D, Schack VR, Amar L, Fischer E et al. Somatic mutations in ATP1A1 and ATP2B3 lead to aldosteroneproducing adenomas and secondary hypertension. Nature Genetics 201345 440-444, 444e441-442. (doi:10.1038/ng.2550)
4 Scholl UI, Goh G, Stolting G, de Oliveira RC, Choi M, Overton JD, Fonseca AL, Korah R, Starker LF, Kunstman JW et al. Somatic and germline CACNA1D calcium channel mutations in aldosteroneproducing adenomas and primary aldosteronism. Nature Genetics 201345 1050-1054. (doi:10.1038/ng.2695)

5 Azizan EAB, Poulsen H, Tuluc P, Zhou J, Clausen MV, Lieb A, Maniero C, Garg S, Bochukova EG, Zhao W et al. Somatic mutations in ATP1A1 and CACNA1D underlie a common subtype of adrenal hypertension. Nature Genetics 201345 1055-1060. (doi:10.1038/ ng.2716)

6 Scholl UI, Stolting G, Nelson-Williams C, Vichot AA, Choi M, Loring E, Prasad ML, Goh G, Carling T, Juhlin CC et al. Recurrent gain of function mutation in calcium channel CACNA1H causes earlyonset hypertension with primary aldosteronism. eLife 20154 e06315. (doi:10.7554/eLife.06315)

7 Beuschlein F, Fassnacht M, Assie G, Calebiro D, Stratakis CA, Osswald A, Ronchi CL, Wieland T, Sbiera S, Faucz FR et al. Constitutive activation of PKA catalytic subunit in adrenal Cushing's syndrome. New England Journal of Medicine 2014370 1019-1028. (doi:10.1056/NEJMoa1310359)

8 Calebiro D, Di Dalmazi G, Bathon K, Ronchi CL \& Beuschlein F. cAMP signaling in cortisol-producing adrenal adenoma. European Journal of Endocrinology 2015173 M99-M106. (doi:10.1530/EJE-150353)

9 Caoili EM, Korobkin M, Francis IR, Cohan RH, Platt JF, Dunnick NR \& Raghupathi KI. Adrenal masses: characterization with combined unenhanced and delayed enhanced CT. Radiology 2002222 629-633. (doi:10.1148/radiol.2223010766)

10 Gomez-Sanchez CE, Qi X, Velarde-Miranda C, Plonczynski MW, Parker CR, Rainey W, Satoh F, Maekawa T, Nakamura Y, Sasano H et al. Development of monoclonal antibodies against human CYP11B1 and CYP11B2. Molecular and Cellular Endocrinology 2014383 111-117. (doi:10.1016/j.mce.2013.11.022)

11 Nanba K, Chen AX, Omata K, Vinco M, Giordano TJ, Else T, Hammer GD, Tomlins SA \& Rainey WE. Molecular heterogeneity in aldosterone-producing adenomas. Journal of Clinical Endocrinology and Metabolism 2016101 999-1007. In press. (doi:10.1210/jc.2015-3239)

12 Pezzi V, Mathis JM, Rainey WE \& Carr BR. Profiling transcript levels for steroidogenic enzymes in fetal tissues. Journal of Steroid Biochemistry and Molecular Biology 200387 181-189. (doi:10.1016/j. jsbmb.2003.07.006)

13 Livak KJ \& Schmittgen TD. Analysis of relative gene expression data using real-time quantitative PCR and the 2(-Delta Delta C(T)) method. Methods 200125 402-408. (doi:10.1006/meth.2001.1262)

14 Larkin SJ, Ferrau F, Karavitaki N, Hernandez-Ramirez LC, Ansorge O, Grossman AB \& Korbonits M. Sequence analysis of the catalytic subunit of PKA in somatotroph adenomas. European Journal of Endocrinology 2014171 705-710. (doi:10.1530/EJE-14-0545)

15 Kuppusamy M, Caroccia B, Stindl J, Bandulik S, Lenzini L, Gioco F, Fishman V, Zanotti G, Gomez-Sanchez C, Bader M et al. A novel KCNJ5-insT149 somatic mutation close to, but outside, the selectivity filter causes resistant hypertension by loss of selectivity for potassium. Journal of Clinical Endocrinology and Metabolism 201499 E1765-E1773. (doi:10.1210/jc.2014-1927)

16 Zheng FF, Zhu LM, Nie AF, Li XY, Lin JR, Zhang K, Chen J, Zhou WL, Shen ZJ, Zhu YC et al. Clinical characteristics of somatic mutations in Chinese patients with aldosterone-producing adenoma. Hypertension 201565 622-628. (doi:10.1161/HYPERTENSIONAHA.114.03346)

Received 22 March 2016

Revised version received 3 May 2016

Accepted 9 May 2016 